\title{
Cholesterol side-chain hydroxylation is associated with expression of P-glycoprotein and disease-free survival in triple negative breast cancer patients
}

\author{
A. Websdale ${ }^{1}$, S.A. Hutchinson ${ }^{1}$, P. Lianto ${ }^{1}$, H. Roberg-Larsen ${ }^{2}$, L.M. Wastall ${ }^{3}$, B. Williams ${ }^{3}$, \\ A. Rose $^{4}$, N. Sharma ${ }^{3}$, T.A. Hughes ${ }^{4}$ and J.L. Thorne ${ }^{1}$ \\ ${ }^{1}$ School of Food Science and Nutrition, University of Leeds, Leeds, LS2 9JT, UK, \\ ${ }^{2}$ Department of Chemistry, University of Oslo, Norway and School of Medicine, \\ ${ }^{3}$ Department of Histopathology, Leeds Teaching Hospitals NHS Trust, LS9 7TF, UK and \\ ${ }^{4}$ School of Medicine, University of Leeds, Leeds, LS9 7TF, UK
}

Triple negative breast cancers (TNBCs) lack cellular protein receptors that can be targeted by the most effective breast cancer drugs. Instead, these patients are treated with non-specific, systemic, cytotoxic chemotherapy agents, such as epirubicin, which can cause severe side effects. Furthermore, chemotherapy resistance is common, rendering many treatments ineffective. P-glycoprotein (Pgp) is a chemotherapy efflux pump that is commonly over-expressed in chemoresistant tumours. However, pharmacological inhibitors of Pgp have typically failed in trials owing to Pgp's crucial role in other tissues; tumour specific mechanisms of Pgp regulation should instead be targeted to induce chemosensitization. Pgp is regulated by cholesterol side-chain hydroxylation products (scOHC) and the liver $\mathrm{x}$ receptor (LXR) in the blood brain barrier ${ }^{(1)}$, potentially linking nutrition and cholesterol metabolism to cellular drug efflux. Interestingly, breast cancer relapse is associated with diets that drive elevated levels of LXR ligands via high circulating cholesterol ${ }^{(2)}$. Our aim was to determine if expression of LXR and synthesis of its scOHC ligands was associated and/or causative of $i$ ) elevated P-gp expression in TNBC, and ii) poor disease-free survival of TNBC patients.

Cancer cell expression of Pgp and scOHC synthesising enzymes (CYP46A1, CH25H, CYP27A1) was assessed with immunohistochemistry (as previously ${ }^{(3)}$ ) using a tissue micro-array containing tissue from 148 TNBC tumours collected at Leeds Teaching Hospitals Trust (ethics reference 06/Q1206/180). In a separate cohort of 31 fresh/frozen TNBC tumours (ethics reference 15/HY/ 0025), we determined: scOHC concentrations for 24OHC, 25OHC, and 26OHC (also known as 27OHC) using LC-MS/MS ${ }^{(4)}$; and Pgp mRNA expression using TaqMan-qPCR (DDcT). In vitro, TNBC cell lines (MDA.MBA.231 and MBA.MB.468) were treated with scOHCs and changes to Pgp mRNA expression assessed by TaqMan. Epirubicin efficacy after scOHC exposure was determined with colony forming assays and MTT. Spearman rank correlation, log-rank Kaplan Meier plots, and one-way ANOVA were used for statistical analysis.

Pgp protein expression in cancer cells in primary tumours positively correlated with $\mathrm{CYP} 46 \mathrm{~A} 1\left(\mathrm{R}^{2}=0.3 ; \mathrm{p}<0.0001\right), \mathrm{CH} 25 \mathrm{H}$ $\left(\mathrm{R}^{2}=0.53\right.$; $\left.\mathrm{p}<0.0001\right)$, and CYP27A1 $\left(\mathrm{R}^{2}=0.07\right.$; $\left.\mathrm{p}<0.01\right)$. High levels of P-gp, CYP46A1 and CH25H (log-rank test: $\mathrm{p}<0.01$ for all) were all associated with reduced disease-free survival. With respect to the scOHCs, 24OHC correlated with P-gp mRNA $\left(\mathrm{R}^{2}=0.5 ; \mathrm{p}=0.04\right)$, but only elevated $25 \mathrm{OHC}$ and $26 \mathrm{OHC}$ associated with reduced disease-free survival $(\mathrm{p}<0.05)$. In vitro, addition of $24 \mathrm{OHC}, 25 \mathrm{OHC}$ or $26 \mathrm{OHC}$ to TNBC cell lines increased P-gp mRNA and reduced epirubicin mediated cytotoxicity in TNBC cells $(\mathrm{p}<0.001)$.

These results suggest that high intratumour oxysterol content is linked to reduced disease-free survival, potentially due to enhanced chemotherapy resistance via Pgp mediated drug efflux. Nutritional and pharmacological reduction of oxysterol levels should be explored as routes to reversing chemoresistance in TNBC.

1. ElAli A \& Hermann DM (2012) Brain Pathol 22, 2, 175-187.

2. Lofterød T et al. (2018) BMC Cancer 18, 654.

3. Kim B et al. (2013) PLoS One 8, 5.

4. Solheim S et al. (2019) J Steroid Biochem Mol Biol 192, 105309. 